\title{
Determination of Nicotine in Tobacco: Collaborative Study*
}

\author{
by \\ James E. Franke ${ }^{1}$, Cliff B. Bennett ${ }^{1}$, Richard E. Davis ${ }^{2}$, Hans V. Thomsen ${ }^{3}$, and Kathleen S. Johnston ${ }^{1}$ \\ ${ }^{1}$ U. S. Tobacco Manufacturing, L. P., Nasbville, TN(USA) \\ ${ }^{2}$ Philip Morris, Research Center, Richmond, VA (USA) \\ ${ }^{3}$ House of Prince, Copenhagen, Denmark
}

\section{SUMMARY}

An international collaborative study was performed to compare several analytical methods for the determination of nicotine in tobacco that are in current use around the world. Five nicotine methods were evaluated and compared, specifically methyl-t-butyl ether (MTBE) extraction with capillary-column gas chromatography (GC), $n$-hexane extraction with capillary-column GC, $n$ hexane extraction with packed-column GC, methanol/ammonia extraction with capillary-column GC, and aqueous extraction with continuous flow analyzer (CFA) colorimetry. A total of 37 laboratories participated in the study, with between 9 and 18 laboratories submitting data per nicotine method. Repeatability, reproducibility, and mean nicotine statistics were calculated and compared for each method. Results for reproducibility (\%) and mean nicotine difference (\%, relative to the mean of the three capillary-GC methods), respectively, for each method are as follows: MTBE method $(2.5 \%,-1.40 \%)$, hexane-capillary $(4.5 \%,+0.06 \%)$, methanol/ammonia $(3.7 \%,+1.34 \%)$, CFA $(4.4 \%,+4.08 \%)$, and hexanepacked $(5.8 \%,+4.14 \%)$. Pair-wise group comparison tests with simultaneous $95 \%$ confidence intervals were used to compare the sample nicotine values between any two given methods. Eight of the ten pair-wise comparisons were statistically different at $95 \%$ confidence, the two statistically indistinguishable pair-wise comparisons being CFA vs. hexane-packed and hexane-capillary vs. methanol/ammonia. The results of this collaborative study will be useful toward the goal of standardizing on a reference method for nicotine analysis in tobacco and tobacco products. [Beitr. Tabakforsch. Int. 19 (2001) 251-265]

\section{ZUSAMMENFASSUNG}

Um mehrere analytische Methoden miteinander zu vergleichen, die gegenwärtig weltweit zur Bestimmung des Nikotingehalts im Tabak angewandt werden, wurde ein internationaler Ringversuch durchgeführt. Fünf Nikotinmethoden wurden ausgewertet und miteinander verglichen, und zwar Methyl-t-butylether (MTBE)-Extraktion mit Kapillar-Gaschromatographie (GC), n-Hexan-Extraktion mit Kapillar-GC, $n$-Hexan-Extraktion mit gepackter Säulen-GC, Methanol/Ammoniak-Extraktion mit Kapillar-GC sowie Wasser-Extraktion mit "Continuous Flow Analysator" (CFA). Insgesamt beteiligten sich 37 Laboratorien an der Studie, wobei pro Nikotinmethode in 9 bis 18 Laboratorien Daten erhoben wurden. Wiederholbarkeit, Reproduzierbarkeit sowie die durchschnittlichen Nikotinwerte wurden berechnet und bei jeder Methode miteinander verglichen. Die Ergebnisse für Reproduzierbarkeit (\%) und die prozentuale Abweichung bezogen auf den Mittelwert der drei Kapillar-GC-Methoden waren für die jeweiligen Methoden wie folgt: MTBE-Methode (2,5\% bzw. $-1,4 \%)$; Hexan-Kapillarsäulenmethode $(4,5 \%$ bzw. $+0,06 \%)$; Methanol/ Ammoniak-Methode (3,7\% bzw. +1,34\%); CFA-Methode (4,4\% bzw. 4,08\% ) und Hexan-Füllkörpersäulenmethode $(5,8 \%$ bzw. $+4,14 \%)$. Anhand paarweiser Gruppenvergleiche und 95\%igen Konfidenzintervallen wurden die Nikotinwerte der Proben von jeweils zwei Methoden miteinander verglichen. Acht der zehn paarweisen Vergleiche ergaben im 95\%-Konfidenzintervall statistisch signifikant unterschiedliche Ergebnisse. Die zwei statistisch nicht unterscheidbaren paarweisen Vergleiche waren CFA-Methode vs. Hexan-Füllkörpersäulenmethode und Hexan-Kapillarsäulenmethode vs. 
Methanol/Ammoniak-Methode. Die Ergebnisse dieses Ringversuchs werden für die Entwicklung einer standardisierten Methode zur Analyse des Nikotins im Tabak und in Tabakprodukten von Nutzen sein. [Beitr. Tabakforsch. Int. 19 (2001) 251-265]

\section{RESUME}

Une étude collective internationale a été effectuée pour comparer plusieurs méthodes analytiques de dosage de la nicotine dans la tabac qui sont en usage actuellement dans le monde entier. Cinq méthodes de dosage de la nicotine ont été évaluées et comparées, à savoir l'extraction par méthyl-t-butyle-éther (MTBE) avec chromatographie en phase gazeuse (CPG) sur colonne capillaire, extraction par $n$-hexane avec CPG sur colonne capillaire, extraction par $n$-hexane avec CPG sur colonne remplie, extraction par méthanol/ammoniac avec CPG sur colonne capillaire et extraction aqueuse avec colorimétrie par analyseur à flux continu (CFA). Au total, 37 laboratoires ont participé à cette étude, et 9 à 18 laboratoires ont fourni des données par méthode de dosage. Pour chaque méthode, la répétabilité, la réproductibilité et les valeurs moyennes de la nicotine ont été calculées et comparées. Les résultats pour la reproductibilité (\%) et la différence moyenne en nicotine (\%, par rapport à la moyenne des trois méthodes CPG-capillaire) sont respectivement pour chaque méthode: méthode MTBE $(2,5 \%$, $-1,40 \%)$; hexane-capillaire $(4,5 \%,+0,06 \%)$; méthanol/ ammoniac $(3,7 \%,+1,34 \%)$; CFA $(4,4 \%,+4,08 \%)$ et hexane-remplie $(5,8 \%, 4,14 \%)$. Des essais de comparaison de groupes appariés avec des intervalles de confiance simultanés de $95 \%$ ont servi à comparer les valeurs de la nicotine des échantillons entre deux méthodes données. Huit des dix comparaisons par paires étaient statistiquement significative au seuil de confiance de $95 \%$, les deux paires statistiquement non discernables étant CFA vs. hexane-remplie et hexane-capillaire vs. méthanol/ammoniac. Les résultats de cette étude collective seront utiles pour la normalisation d'une méthode de référence pour l'analyse de la nicotine dans le tabac et les produits du tabac. [Beitr. Tabakforsch. Int. 19 (2001) 251-265]

\section{INTRODUCTION}

Quantification of nicotine in tobacco and tobacco products has been and continues to be important in the areas of science, industrial manufacturing, and government regulatory issues. Given the wide range of interest in nicotine quantification and the many industries and agencies responsible for reporting and monitoring nicotine levels, many analytical methods for the determination of nicotine have emerged in the last 50 years. BORGERDING et. al. (1) have identified 14 different methods for nicotine analysis that have developed since 1950, and the authors point out that these developments largely parallel the technological advances in analytical instrumentation. As a result of this proliferation of methods for nicotine analysis, a variety of analytical methods are in current use. In addition, a variety of solvents are in current use to quantitatively extract nicotine from tobacco, including methyl-t-butyl ether (MTBE), $n$-hexane, methanol/ ammonia, or aqueous solvents. The range in solvent extraction systems has emerged in the past from the variety of sample matrices encountered in the categories of tobacco and tobacco products. Extraction methods have evolved for particular classes of tobacco samples to optimize the extraction of nicotine and to minimize the extraction of potential interferences (e.g., flavorings, humectants).

Given the existence of multiple methods for nicotine analysis in tobacco, government regulatory agencies have specified a variety of methods for various tobacco industries or products. A number of government reporting requirements for tobacco and tobacco products specify use of the MTBE extraction method published in the Federal Register (2). Some US states have accepted use of continuous flow analysis (CFA) and aqueous extraction (3) for nicotine determination. Meanwhile, some cigarette manufacturers have adopted methanol/ammonia extraction and capillary-column GC for determining nicotine in their cigarette products. Still other nicotine methods are used in Germany based on $n$-hexane extraction with either capillary- or packed-column GC.

The current paper presents an international collaborative study on the quantitative determination of nicotine in tobacco and tobacco products. The study was defined, initiated, and managed by cooperation of the Nicotine Subcommittee of the Tobacco Science Research Conference Analytical Methods Committee (TSRC-AMC), the Routine Analytical Chemistry Sub Group of the Cooperation Centre for Scientific Research Relative to Tobacco (CORESTA), and Deutsches Institut für Normung (DIN). The goal of the study was to compare several nicotine methods that are in current use, specifically those using MTBE, $n$-hexane, methanol/ammonia, and aqueous (CFA) extractions. The repeatability and reproducibility of each method were compared, and agreement in determined nicotine concentration among the methods was assessed. A wide range of tobacco sample types was used in the study to identify a dependence (if any) of nicotine determination on sample type for any of the methods. The results from this study are intended to provide information and to encourage discussion on the possibility of adopting a reference analytical method for determining nicotine in tobacco and tobacco products.

\section{MATERIALS AND METHODS}

A total of 37 laboratories, representing CORESTA, DIN, and TSRC-AMC, participated in the study. Of the 37 laboratories, 18 provided data on the MTBE method, 14 on the hexane-capillary method, 17 on the methanol/ 
Table 1.

Characteristics of the 13 tobacco samples distributed to laboratories in the collaborative study ${ }^{a}$

\begin{tabular}{|c|c|c|c|c|c|}
\hline Sample & Description & Category & $\begin{array}{l}\% O \mathrm{OV} \sim 50 \mathrm{~g} \\
\text { sample size }\end{array}$ & $\begin{array}{l}\% O V \sim 15 \mathrm{~g} \\
\text { sample size }\end{array}$ & Difference \\
\hline 1 & Skoal Longcut & Smokeless & 53.89 & 53.41 & 0.48 \\
\hline 2 & Rooster & Smokeless & 54.99 & 54.69 & 0.30 \\
\hline 3 & Kodiak & Smokeless & 53.69 & 53.79 & -0.10 \\
\hline 4 & Cigar & Cigar & 13.60 & 13.30 & 0.30 \\
\hline 5 & Redman loose leaf & Smokeless & 24.29 & 23.64 & 0.65 \\
\hline 6 & Timberwolf & Smokeless & 51.10 & 50.63 & 0.47 \\
\hline 7 & Marlboro & Cigarette & 13.31 & 13.22 & 0.10 \\
\hline 8 & Salem & Cigarette & 14.25 & 14.02 & 0.22 \\
\hline 9 & Burley leaf & Leaf & 11.92 & 11.84 & 0.08 \\
\hline 10 & Oriental & Leaf & 10.68 & 10.46 & 0.22 \\
\hline 11 & Bright leaf & Leaf & 11.82 & 11.65 & 0.17 \\
\hline 12 & Pipe tobacco & Pipe & 28.10 & 27.92 & 0.19 \\
\hline \multirow[t]{2}{*}{13} & 1R4F & Cigarette & 14.05 & 13.87 & 0.19 \\
\hline & & \multicolumn{3}{|c|}{ Average $\%$ difference } & 0.25 \\
\hline
\end{tabular}

\footnotetext{
${ }^{\text {aT }}$ The laboratory that distributed samples to the study participants determined the percent oven volatiles (\%OV) content of each sample. Most participants received approximately $50 \mathrm{~g}$ sample sizes in the initial distribution. The remaining participants received approximately $15 \mathrm{~g}$ sample sizes in a later distribution.
}

ammonia method, 15 on the CFA method, and nine on the hexane-packed method.

Each participating laboratory in the study was given 13 tobacco samples to analyze using as many of the methods as possible. The 13 samples provided to participants are described in Table 1, and they represent several categories of tobacco samples, including cigarette filler, leaf, smokeless products, pipe tobacco, and cigar. The cigarette, leaf, and loose leaf samples were ground to pass a four millimeter screen prior to distribution. The cigar sample was ground to pass a two millimeter screen, and the pipe tobacco and moist snuff samples were distributed as received from the manufacturers. Participating laboratories in the collaborative study were instructed to analyze the samples without any additional physical preparation of the samples. Most labs received approximately $50 \mathrm{~g} \mathrm{sam}$ ples, but a distribution of samples later in the study was of necessity smaller in size (approximately $15 \mathrm{~g}$ ). The smaller-size samples possessed slightly lower \% ovenvolatiles (\%OV) content (on average) than the larger-size samples, as shown by the $\% \mathrm{OV}$ values in Table 1 . The laboratory responsible for distributing samples to all participants in the collaborative study provided the $\% \mathrm{OV}$ measurements shown in Table 1 . The mean values of the two \%OV measurements for each sample in Table 1 were used to convert wet-weight-basis nicotine values that were reported by participants to a dry-weight basis. Three extractions, with duplicate measurements per extraction, were required per sample per method for a total of 78 measurements per method. Each laboratory was given a nicotine salicylate standard sample for preparing standard solutions to calibrate the $n$-hexane and CFA methods. For MTBE and methanol/ammonia methods, each laboratory was instructed to use its own source of nicotine standard to prepare calibration solutions for these methods.

\section{MTBE method (capillary-column GC)}

In the MTBE method (4), $7 \mathrm{~mL}$ of $5 \mathrm{~N} \mathrm{NaOH}(5 \mathrm{~mL}$ of $2 \mathrm{~N} \mathrm{NaOH}$ specified in CDC method [2]) is mixed with $1 \mathrm{~g}$ tobacco sample in a bottle and the mixture is allowed to stand $15 \mathrm{~min}$. After this time, $50 \mathrm{~mL}$ of MTBE extraction solution with quinoline internal standard is added and the bottles are capped tightly. The bottles are shaken horizontally for $3 \mathrm{~h}$ and when the phases separate $(\leq 24 \mathrm{~h})$ aliquots are taken and placed in autosampler vials for analysis with capillary GC. The GC response is calibrated using a series of nicotine standard solutions ranging from 0.1 to $0.8 \mathrm{mg} / \mathrm{mL}$ of nicotine in MTBE solution. The nicotine standard and tobacco extract solutions are made to contain quinoline internal standard (IS) at $0.4 \mathrm{mg} / \mathrm{mL}(0.04 \%)$. The GC uses a flame ionization detector and a DB-5 column $(30 \mathrm{~m} \times 0.32 \mathrm{~mm}$ i.d. $)$ at 1 $\mu \mathrm{m}$ film thickness $(0.2 \mu \mathrm{m}$ to $0.5 \mu \mathrm{m}$ specified in CDC method [2]). The GC oven temperature program for sample analysis is $10^{\circ} \mathrm{C} / \mathrm{min}$ between $110-185^{\circ} \mathrm{C}$ and $6^{\circ} \mathrm{C} / \mathrm{min}$ between $185-245^{\circ} \mathrm{C}$, with 10 min hold at $250^{\circ} \mathrm{C}$ and 5 min equilibration at $110^{\circ} \mathrm{C}$. The chromatographic conditions specify detector temperature of $250^{\circ} \mathrm{C}$, injector temperature of $250^{\circ} \mathrm{C}$, carrier gas flow rate of $1.7 \mathrm{~cm}^{3} / \mathrm{min}$ at $100{ }^{\circ} \mathrm{C}$, a splitless injection (split injection specified in CDC method [2]), and injection volume of $1 \mu \mathrm{L}$. The nicotine-to-quinoline peak area ratio is used for all tobacco extract and nicotine standard solutions to compensate for any changes in GC response. 
This method differs in minor details, as noted above, from the extraction and chromatographic analysis specified for CDC reporting of nicotine in smokeless tobacco in the USA (2).

\section{n-Hexane method (capillary-or packed-column GC)}

For the $n$-hexane methods (5), nicotine extraction is accomplished by mixing $1 \mathrm{~g}$ of tobacco sample with $20 \mathrm{~mL}$ of water, $10 \mathrm{~mL}$ conc. $\mathrm{NaOH}(32 \%)$, and $40 \mathrm{~mL}$ of $n$ hexane extraction solution with $n$-heptadecane IS $(0.5$ $\mathrm{mg} / \mathrm{mL}$ or $0.05 \%)$. This mixture is shaken for $1 \mathrm{~h}$ in such a way that the phases are well mixed, after which aliquots are taken for GC analysis. The GC response for the $n$-hexane methods is calibrated using a series of nicotine standard solutions ranging from 0.12 to $0.97 \mathrm{mg} / \mathrm{mL}$ of nicotine in $n$-hexane solution, which corresponds to $0.5 \%$ to $3.0 \%$ nicotine in tobacco. The GC uses a flame ionization detector and either a packed or capillary column. The packed column is $1.5-2.0 \mathrm{~m} \times 2.0-4.0 \mathrm{~mm}$ i.d. with a stationary phase of $10 \%$ polyethylene glycol (PEG) $20000+2 \% \mathrm{KOH}$ on acid washed support material (80-100 mesh). Packed column chromatographic conditions specify detector and injector temperatures of $270^{\circ} \mathrm{C}$, oven temperature of $170^{\circ} \mathrm{C}$, injection volume of $1 \mu \mathrm{L}$, and carrier gas of $30 \mathrm{~mL} / \mathrm{min}$ helium, $30 \mathrm{~mL} / \mathrm{min}$ hydrogen, or $400 \mathrm{~mL} / \mathrm{min}$ air. The capillary GC method uses a fused silica column with $0.15 \mathrm{~mm}$ i.d. or megabore $0.53 \mathrm{~mm}$ i.d. (e.g., DB-17 or SE 54) and $1 \mu \mathrm{m}$ film thickness. Capillary-column chromatographic conditions specify a 10:1 split flow, detector and injector temperatures of $270{ }^{\circ} \mathrm{C}$, oven temperature of $170{ }^{\circ} \mathrm{C}$, injection volume of $1 \mu \mathrm{L}$, and carrier gas of $5 \mathrm{~mL} / \mathrm{min}$ helium (or nitrogen), $30 \mathrm{~mL} / \mathrm{min}$ hydrogen, or $400 \mathrm{~mL} / \mathrm{min}$ air (5-25 mm/min nitrogen make-up gas). The nicotine-to- $n$ heptadecane peak area ratio is used for all tobacco extract and nicotine standard solutions to compensate for any changes in GC response.

\section{Methanol/ammonia method (capillary-column GC)}

In the methanol/ammonia extraction method (6), nicotine extraction proceeds by mixing $2 \mathrm{~mL}$ of $20 \%(v / v)$ ammonium hydroxide with $2 \mathrm{~g}$ tobacco sample in a flask, capping the flask, and allowing it to stand $30 \mathrm{~min}$. After this time, $50 \mathrm{~mL}$ of methanol extraction solution with isoquinoline internal standard is added and the flasks are shaken at $270-275 \mathrm{r} / \mathrm{min}$ for $1 \mathrm{~h}$. Aliquots are taken and analyzed with capillary-column GC. The GC response is calibrated using a series of nicotine standard solutions ranging from 0.1 to $1.2 \mathrm{mg} / \mathrm{mL}$ of nicotine in methanol/ammonia solution. The nicotine standard and tobacco extract solutions are made to contain $0.05 \%$ $(v / v)$ isoquinoline IS and $0.8 \%(v / v)$ of ammonium hydroxide pre-treatment solution. The GC uses a StabilwaxDB column, $15 \mathrm{~m} \times 0.25 \mathrm{~mm}$ i.d. and $0.25 \mu \mathrm{m}$ film thickness. The GC oven temperature program for sample analysis is $5^{\circ} \mathrm{C} / \mathrm{min}$ between $150-200{ }^{\circ} \mathrm{C}$ with $0 \mathrm{~min}$ final time and 0 min equilibration time. Chromatographic conditions specify a detector temperature of $250^{\circ} \mathrm{C}$, injector temperature of $225{ }^{\circ} \mathrm{C}$, oven temperature of $150^{\circ} \mathrm{C}$ with linear velocity of $42 \mathrm{~cm} / \mathrm{sec}$ and split flow of $34 \mathrm{~mL} / \mathrm{min}$, and injection volume of $1 \mu \mathrm{L}$. The nicotine-to-isoquinoline peak area ratio is used for all tobacco extract and nicotine standard solutions to compensate for any changes in GC response.

\section{Aqueous continuous flow analysis method}

The aqueous extraction method uses CFA to assay total alkaloids in aqueous tobacco extracts. The total alkaloid content of the extract is determined by reaction with sulphanilic acid and cyanogen chloride, the latter being generated in situ by reaction of potassium cyanide and chloramine $\mathrm{T}$ solution (3). Nicotine standard solutions are prepared from nicotine hydrogen tartrate (NHT) in the range of 0.04 to $0.8 \mathrm{mg} / \mathrm{mL}$ nicotine in aqueous solution, taking into consideration the purity of the NHT standard. The nicotine standards are passed through the CFA and a calibration curve is built from colorimetric measurements at $460 \mathrm{~nm}$ in a $2 \mathrm{~mm}$ path optical cell. Aqueous extracts of tobacco are prepared by mixing 250 $\mathrm{mg}$ tobacco samples with $25 \mathrm{~mL}$ distilled water followed by shaking each mixture for $30 \mathrm{~min}$. After filtering mixtures (Whatman no. 40 filter), filtrates are measured with the CFA analyzer at $460 \mathrm{~nm}$, and total alkaloids are assayed by comparison to the nicotine calibration curve.

\section{STATISTICAL METHODS}

The International Organization for Standardization (ISO) document 5725-2 (7) specifies the method used in this study to identify outliers in a given data set and to calculate repeatability $\left(s_{r}\right)$ and reproducibility $\left(s_{R}\right)$ statistics for the data set once outliers are removed. Removal of outlying samples is required if a data set is to have normally distributed mean nicotine values at each level and homogeneity of variances among laboratories at each level. Normality and homogeneity are necessary for the reliable determination of repeatability and reproducibility statistics for each analytical method that is evaluated. Outliers were determined for each method in the collaborative study based on Cochran, Grubbs, and Mandel $b$ and $k$ statistics specified in ISO 5725-2. However, certain decisions are left to the judgment of the analyst, such as the number of times to apply Cochran's test and whether to discard all data from a laboratory if a majority of its samples are flagged as outliers. In these cases, our best judgment was applied to avoid being too aggressive yet not too conservative in our approach to outlier removal. As a guideline to outlier removal, the Association of Official Analytical Chemists (AOAC) recommends that no more than $2 / 9$ of the data for a method should be discarded; otherwise, the applicability of the method should be considered (8). Following outlier removal, 
repeatability and reproducibility statistics are calculated for each method separately using analysis of variance (ANOVA) principles.

In ISO 5725-2, the repeatability variance of the $j^{\text {th }}$ tobacco sample, $s_{r j}^{2}$, is the weighted average variance of the set of triplicate extraction measurements of all laboratories analyzing the $j^{\text {th }}$ sample using a given nicotine method. $s_{r j}^{2}$ is calculated using formula [20] in ISO 5725-2, and it is weighted according to the number of degrees of freedom for each sample (variances determined from more replicates are given more weight). $s_{r j}^{2}$ represents the random experimental error associated with each individual nicotine measurement.

The between-laboratory variance of the $j^{\text {th }}$ tobacco sample, $s_{\mathrm{L} j}^{2}$, is the weighted average variance of the mean nicotine determination for the $j^{\text {th }}$ sample from all laboratories analyzing that sample (formulas [19]-[23] in ISO $5725-2) . s_{\mathrm{L} j}^{2}$ isolates the variance in mean values by subtracting $s_{r j}^{2} / n$ from the total variance in the mean values (repeatability variance of $s_{r j}^{2}$ is present in each individual nicotine measurement and mean repeatability variance of $s_{r j}^{2} / n$ is present in each mean value). The division by $n$ is done because the repeatability variance is random and $n$ replicate nicotine measurements of the same sample are made by each laboratory to calculate a mean nicotine value. Hence, the contribution of $s_{r j}^{2}$ to the mean values for the $j^{t h}$ sample is reduced to $s_{r j}^{2} / n$ (ISO 5725-2 shows how to calculate a weighted $n$ [formula 23] if not all laboratories provided the same number of replicate measurements). $s_{\mathrm{L} j}^{2}$ is the variance about the general mean nicotine determined for the $j^{\text {th }}$ sample using a given nicotine method. The general mean nicotine (formula [19] in ISO 5725-2) is the weighted mean nicotine concentration determined from the mean nicotine measurements from all laboratories analyzing the $j^{\text {th }}$ sample using a given nicotine method. $s_{\mathrm{L} j}^{2}$ is a weighted average variance because it weights the mean values by the number of replicates used to calculate each mean nicotine value by each laboratory (mean nicotine values determined from more replicates receive more weight).

The reproducibility variance, $s_{R j}^{2}$ is simply the sum of the repeatability variance and the between-laboratory variance for the $j^{\text {th }}$ sample using a given nicotine analytical method. Using these statistical computations, the repeatability and reproducibility variances and the mean nicotine concentrations were obtained for each of the 13 samples for each of the five nicotine analytical methods. The five nicotine methods were compared on the basis of these variance statistics and mean nicotine values.

\section{RESULTS AND DISCUSSION}

The nicotine collaborative study was designed to determine and to compare the precision of multiple analytical methods for the determination of nicotine in tobacco and tobacco products. The samples distributed to partici- pants varied in both nicotine and type of tobacco sample (i.e., sample matrix). Thus, the primary effect studied in the collaborative study was the effect of varying the method for nicotine determination; but a secondary effect influencing the results was the effect of sample matrix since each of the 13 tobacco samples represents a different sample matrix.

\section{Dry-weight basis nicotine values}

The list of samples distributed to participants and the $\% \mathrm{OV}$ content of each sample are detailed in Table 1 . The samples span a wide range in \%OV values $(11-55 \% \mathrm{OV})$ due to the variety of tobacco sample categories included in the study. The range of nicotine spanned by the 13 tobacco samples was 6.8 to $37.2 \mathrm{mg} / \mathrm{g}$ nicotine (wetweight basis) or 9.0 to $42.7 \mathrm{mg} / \mathrm{g}$ nicotine (dry-weight basis). While the range of variation of wet- or dry-weight basis values is similar, the distribution of each is quite different due to variation in the $\% \mathrm{OV}$ of the samples. If reported on a wet-weight basis, ten samples would span a narrow range of nicotine (10.5 to $17.6 \mathrm{mg} / \mathrm{g}$, wet), two would show much higher nicotine (31.7 and $37.2 \mathrm{mg} / \mathrm{g}$, wet) and one would show lower nicotine $(6.8 \mathrm{mg} / \mathrm{g}$, wet). In contrast, the dry-weight basis nicotine values are more evenly distributed over their range of nicotine variation than the corresponding wet-weight basis nicotine values.

The collaborative study data were evaluated using dryweight-basis nicotine values in order to remove the influence of variable \%OV in the samples. Use of either dry-weight-basis or wet-weight-basis nicotine values yields the same average percent repeatability and average percent reproducibility results, since the same \%OV value was applied to each laboratory's reported wetweight values for a given sample. The use of dry-weight nicotine values does, however, influence the regression of repeatability or reproducibility values on the mean nicotine values for each sample because the dry-weight-basis nicotine values are more evenly distributed over the range of nicotine variation than are the wet-weight-basis values.

The oven volatile data in Table 1 also show that the $50 \mathrm{~g}$ samples have slightly more oven volatiles on average $(0.25 \%)$ than the $15 \mathrm{~g}$ samples prior to their distribution to participants. Since the laboratories reporting the hexane-capillary method received predominantly the $15 \mathrm{~g}$ samples, their reported nicotine values may be no more than $0.09 \mathrm{mg} / \mathrm{g}(0.39 \%)$ higher on average than values reported from laboratories using the $50 \mathrm{~g}$ samples. The $0.39 \%$ value represents an upper limit of possible bias for laboratories analyzing the $15 \mathrm{~g}$ samples. None of the collaborative study data were adjusted for small differences in \%OV because detailed \%OV data would be required on each sample from each laboratory to adjust the original data correctly. It should be noted that even if the bias were as large as the upper limit estimate of $0.39 \%$ the conclusions of this study would not be altered. 
A nicotine salicylate (NS) standard material was distributed to each participant with the intention that it be used for all methods. Testing of this reference material indicated that its solubility in MTBE and methanol could vary without uniform handling procedures. Therefore, the final instructions for the MTBE and methanol/ammonia methods specified that participants use their own nicotine standard materials and provide purity analysis if possible. Six laboratories submitted a total of 9 purity determinations of the NS standard, and six laboratories submitted a total of 11 determinations of their own nicotine standards. After outlier testing, the average purity of the NS standard was $98.6 \%$, while the other nicotine standards (most often certified standards, or lab-distilled) averaged $99.3 \%$.

The possible effect of the NS standard purity on nicotine determinations could be investigated further within the hexane-capillary data set, in which seven of the laboratories used the NS standard and seven used their own nicotine standards. A consistent effect on nicotine values from differences in the purity of the nicotine standards should become manifest as a multiplicative bias (i.e., nonunity slope) between the two sets of hexane-capillary data. A linear least-squares regression of the nicotine values of the 13 tobacco samples obtained using lab-specific nicotine standards, $n_{\mathrm{Lab}}$, on the nicotine values of the same 13 samples obtained using the NS standard, $n_{\mathrm{NS}}$, yielded $n_{\mathrm{Lab}}=1.018 \times n_{\mathrm{NS}}$ with $R^{2}=0.9979$ (using dryweight-basis nicotine values). This result indicates a weak effect, if any, on nicotine values in the two hexane-capillary subsets resulting from differences in purity of the standards (1.8\% slope). Given insufficient evidence to support a significant effect from nicotine standard purity, none of the reported nicotine measurements in the collaborative study were adjusted for the purity of the nicotine standards.

\section{Special treatment of $\mathrm{n}$-hexane method data}

The $n$-hexane method allows the analyst to choose either a packed or capillary column; and as a result, about $40 \%$ of the labs reporting $n$-hexane data used a packed column while $60 \%$ used a capillary column. We decided to treat these two data sets as independent nicotine methods because the analytical technique used in each differs (packed vs. capillary column) and because corresponding sample nicotine values in each differed by a multiplicative bias. The magnitude of this multiplicative bias was estimated to be $4.5 \%$ (hexane-packed values being greater) from the slope of the linear least-squares regression of the mean nicotine values for hexane-packed, $n_{\text {packed }}$, vs. hexane-capillary, $n_{\text {cap }}$. The regression analysis yielded $n_{\text {packed }}=1.0446 \times n_{\text {cap }}-0.0997$ with $R^{2}=0.9993$ (using dry-weight basis nicotine values). Based on these observations, the hexane data were treated as two independent methods, hexane-capillary and hexane-packed.
The data received from the 37 participating laboratories resulted in a total of 5,289 nicotine measurements on the 13 tobacco samples, of which $97.3 \%$ were in compliance with the study directives. The non-compliant $2.7 \%$ of values were discarded at the outset prior to assessing the data for outliers and subsequent statistical evaluation of the collaborative study data. The two conditions that resulted in non-compliant measurements were the use of the wrong type of GC column and insufficient number $(<3)$ of nicotine extractions per sample.

The outlier detection and removal process detailed in ISO 5725-2 was applied to the compliant measurements from each of the five nicotine methods separately. For the MTBE method, all data from one laboratory was removed at the outset (non-compliant) because it was not obtained using a capillary column as required by the study directives for the MTBE method. Outlier analysis identified that $16.3 \%$ of the data in the remaining 17 laboratories should be excluded, and these data were removed prior to calculating final mean nicotine values and repeatability and reproducibility statistics for each sample. The MTBE data set is a good example of how the ISO outlier procedures require interpretation and judgment by the analyst. ISO 5725-2 specifies a protocol for removing outliers based on Cochran and Grubbs statistics, stating that single-sample Grubbs outlier tests should be applied prior to double-sample Grubbs outlier tests. ISO 5725-2 also recommends that the analyst use Mandel $b$ and $k$ plots to check for consistency among all laboratories. The Mandel $b$ plot for the MTBE data clearly showed that two laboratories reported consistently lower nicotine values relative to the other 15 laboratories reporting MTBE data. Based on the Mandel $h$ plot, the double-sample Grubbs outlier tests were applied prior to the singlesample Grubbs outlier tests, deviating slightly from the ISO protocol. The double-sample Grubbs outlier tests revealed that 11 of the 13 pairs of samples from these two laboratories were outliers at $99 \%$ confidence, and these 22 values were deleted. No further deviation from the ISO 5725-2 outlier protocols was necessary. Application of the Cochran test for variance outliers identified 8 of the 13 samples in a third laboratory as outliers at $99 \%$ confidence, and these 8 values were deleted. Only 6 additional values were deleted as outliers at $99 \%$ confidence, meaning that $83 \%$ of all ISO $5725-2$ outliers detected in the MTBE data derived from just three of the 17 laboratories reporting compliant MTBE data.

The ISO outlier protocol was repeated similarly for the hexane-capillary data set, in which $6.6 \%$ of its data were determined to be outliers. For the remaining nicotine methods, $12.8 \%$ of the methanol/ammonia, $16.8 \%$ of the CFA, and $18.0 \%$ of the hexane-packed data, respectively, were deleted as outliers. Less than 2/9 of the data were removed as outliers for all methods, as recommended by the AOAC guidelines (8). The data deleted using the ISO procedures are significantly outlying samples, since the 
Table 2.

Mean nicotine for each tobacco sample and each analytical method, reported on a dry-weight basis (i.e., weight of nicotine per weight of dried sample)

\begin{tabular}{l|r|r|r|r|r}
\hline \multirow{2}{*}{ Sample } & \multicolumn{5}{|c}{ Mean nicotine $(\mathrm{mg} / \mathrm{g}$, dry basis) } \\
\cline { 2 - 6 } & MTBE & $\begin{array}{l}\text { Hexane- } \\
\text { capillary }\end{array}$ & $\begin{array}{l}\text { Methanol/ } \\
\text { ammonia }\end{array}$ & CFA & $\begin{array}{r}\text { Hexane- } \\
\text { packed }\end{array}$ \\
\hline 1 & 28.4 & 29.3 & 29.1 & 29.5 & 30.4 \\
2 & 25.9 & 26.4 & 26.1 & 27.1 & 27.5 \\
3 & 22.9 & 23.1 & 23.3 & 23.4 & 23.6 \\
4 & 13.3 & 13.6 & 13.5 & 14.3 & 14.0 \\
5 & 8.5 & 8.8 & 8.8 & 9.2 & 9.3 \\
6 & 30.7 & 31.5 & 30.9 & 31.7 & 32.6 \\
7 & 18.8 & 19.0 & 19.6 & 20.0 & 19.7 \\
8 & 18.6 & 18.5 & 19.4 & 19.8 & 19.3 \\
9 & 41.2 & 41.3 & 42.4 & 42.8 & 43.5 \\
10 & 12.7 & 12.7 & 13.1 & 14.1 & 13.4 \\
11 & 35.2 & 35.7 & 35.3 & 36.4 & 37.4 \\
12 & 14.1 & 14.2 & 14.9 & 15.2 & 15.0 \\
13 & 20.0 & 20.2 & 20.8 & 20.9 & 20.7 \\
\hline
\end{tabular}

ISO 5725-2 outlier tests require quite stringent $99 \%$ confidence in general for exclusion (stragglers at $95 \%$ confidence are removed if they can be explained). Where analyst judgment was required in removing outliers, such judgment was made based on rational consideration of relationships and patterns in the data, as in the MTBE analysis discussed above. Such a considered approach was used because removal of outliers is critical to obtain valid estimates of mean nicotine values as well as repeatability and reproducibility statistics for each method. Outliers, if present, would invalidate the assumptions about the normality of the population variance and the assumption of homogeneity of variance, as discussed in the Statistical Methods section.

Several factors existed in the collaborative data set that likely induced additional, uncontrolled variance into the data set, which could affect the number of samples flagged as outliers for any of the nicotine method data sets. For example, it is possible that the samples used by each laboratory had slightly different \%OV content caused by differences in environmental exposure and sample handling. This condition would lead to additional betweenlaboratory variance and possibly more outlier samples detected. Since laboratories were instructed to use their own nicotine standards for some of the nicotine methods, any differences in the purity of their standards would also lead to increased between-laboratory variance and possibly more outlier samples detected. Finally, most laboratories participating in the study do not routinely use all of the five nicotine methods included in the collaborative study. Some laboratories will likely produce more reproducible results for some methods but not for other methods depending on their level of experience with each of the methods. This situation could create
Table 3.

Relative mean nicotine values (normalized to the mean of the three capillary GC methods for each sample)

\begin{tabular}{l|c|c|c|c|c}
\hline \multirow{2}{*}{ Sample } & \multicolumn{6}{|c}{ Relative mean nicotine (\%, dry basis) for each method } \\
\cline { 2 - 6 } & MTBE & $\begin{array}{c}\text { Hexane- } \\
\text { capillary }\end{array}$ & $\begin{array}{c}\text { Methanol/ } \\
\text { ammonia }\end{array}$ & CFA & $\begin{array}{c}\text { Hexane- } \\
\text { packed }\end{array}$ \\
\hline 1 & 0.982 & 1.013 & 1.005 & 1.019 & 1.050 \\
2 & 0.992 & 1.008 & 1.000 & 1.038 & 1.050 \\
3 & 0.991 & 1.000 & 1.009 & 1.011 & 1.019 \\
4 & 0.988 & 1.010 & 1.002 & 1.057 & 1.036 \\
5 & 0.975 & 1.013 & 1.013 & 1.054 & 1.073 \\
6 & 0.988 & 1.016 & 0.996 & 1.021 & 1.049 \\
7 & 0.981 & 0.994 & 1.024 & 1.044 & 1.031 \\
8 & 0.985 & 0.983 & 1.032 & 1.051 & 1.026 \\
9 & 0.989 & 0.992 & 1.019 & 1.027 & 1.043 \\
10 & 0.991 & 0.992 & 1.018 & 1.098 & 1.044 \\
11 & 0.994 & 1.008 & 0.998 & 1.028 & 1.056 \\
12 & 0.979 & 0.986 & 1.035 & 1.057 & 1.044 \\
13 & 0.982 & 0.994 & 1.023 & 1.026 & 1.017 \\
\hline
\end{tabular}

occurrences of higher reproducibility variance or even systematic errors in mean nicotine for some laboratories relative to others, leading to more outlier samples.

\section{Comparison of methods based on mean nicotine}

Each nicotine method data set was analyzed separately. After outlier detection and removal in each data set, mean nicotine values for each of the 13 tobacco samples were calculated. The mean nicotine values calculated for all samples and methods are detailed in Table 2. The table shows that in the 13 tobacco samples nicotine ranges between about 9 and $43 \mathrm{mg} / \mathrm{g}$ (dry-weight basis). Table 3 provides the relative mean nicotine values of each of the 13 samples for each method, which are calculated relative to the mean nicotine for each sample derived from the three capillary GC methods. The mean of the three capillary GC methods was chosen as the reference for the relative mean calculations because these three techniques use similar analytical instrumentation and their mean nicotine values are more similar to each other than they are to the CFA or hexane-packed method mean nicotine values. Table 7 summarizes the difference in mean nicotine values among all five methods, relative to the mean of the three capillary GC methods. The CFA and hexane-packed methods have mean nicotine values that are $4.1 \%$ higher on average than the mean values of the three capillary GC methods, which differ at most by $2.7 \%$ on average. The relative differences in mean nicotine are graphically displayed in Figure 1. For each mean percent difference bar, $m$, the error bar represents the estimate of the standard error on the relative mean for each method. 


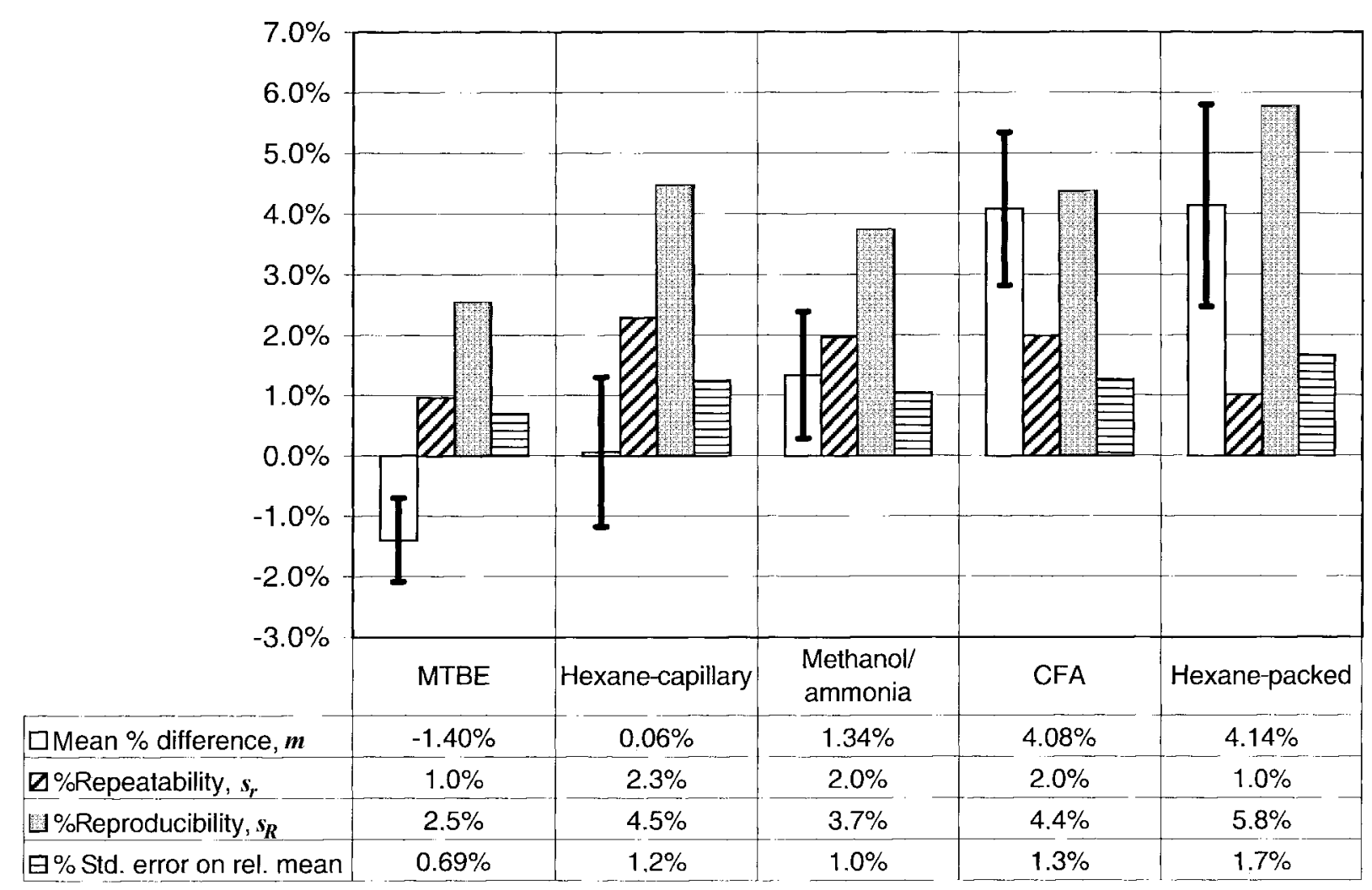

Figure 1.

Mean differences, repeatability, and reproducibility of nicotine methods; percent standard error on the relative mean (last row of values) is calculated from $\pm(1+m) s_{R} / \sqrt{13}$ (mean \% differences are relative to mean of the three capillary GC methods)

Two-way ANOVA was used to determine that the differences in mean nicotine among the five methods were statistically significant. Pair-wise group comparisons and simultaneous $95 \%$ confidence intervals were used to determine what specific nicotine methods were or were not statistically indistinguishable, and the results are shown in Table 4. The pair-wise comparisons given in Table 4 show that the hexane-capillary method is statistically indistinguishable from the methanol/ammonia method and that CFA method is statistically indistinguishable from the hexane-packed method.

The relative mean sample values (normalized to the mean of the three capillary methods) are compared by sample category in Figure 2. When the cigarette filler samples are considered alone, the hexane-packed, CFA, and methanol/ammonia methods yield $4.4 \%$ higher nicotine values on average than do the hexane-capillary and MTBE methods. However, when all sample types are considered the hexane-packed and CFA methods yield $4.1 \%$ higher values on average than the three capillary GC methods. Overall, then, the CFA and hexane-packed methods seem to yield nicotine values that are biased high by about $4 \%$. Because the same extraction solvent is used in the hexane-packed and hexane-capillary methods, the positive bias between these two hexane-based methods cannot be ascribed to a difference in extraction efficiency of the solvent. The positive bias of the hexane- packed method is likely caused by unresolved peak interferences caused by the poorer peak resolution in packedcolumn chromatography (9). The CFA method may be biased high due to reported interferences from other tobacco alkaloids, such as nornicotine (1).

\section{Calculation of repeatability and reproducibility statistics}

For each of the 13 samples in each of the five nicotine methods, repeatability, $s_{r}$, and reproducibility, $s_{R}$, statistics were calculated (after outlier removal). An iterative weighted least-squares (WLS) regression (7) of repeatability values $\left(s_{r}\right)$ on mean nicotine values, $m_{\mathrm{Nic}}$ was done for each method to determine if $s_{r}$ was correlated to $m_{\mathrm{Nic}}$. The same regression test was done to determine if the reproducibility values $\left(s_{R}\right)$ were related to $m_{\text {Nic }}$ values for each method. The iterative WLS regression is initialized with weights for each sample, $w_{j}$, given by $1 / s_{r j}^{2}$ for repeatability or $1 / s_{R j}^{2}$ for reproducibility. Upon convergence, WLS regression produces the weighted least squares estimates of the intercept $\left(\hat{b}_{w, r}\right.$ or $\left.\hat{b}_{w, R}\right)$ and slope $\left(\hat{m}_{w, r}\right.$ or $\left.\hat{m}_{w, R}\right)$ of the best-fit line relating $s_{r j}$ or $s_{R j}$ to $m_{\mathrm{Nic}}$. If a satisfactory regression result is obtained, then $\hat{m}_{w, r}$ or $\hat{m}_{w, R}$ is used as the best estimate, respectively, of $s_{r}$ or $s_{R}$ for a given nicotine method (7). If there is no significant correlation with $m_{\mathrm{Nic}}$, then the best-fit 
Table 4.

Pair-wise group comparisons of mean nicotine values among all five analytical methods using simultaneous $95 \%$ confidence intervals

\begin{tabular}{lll|c|c|c}
\hline Result & First group & Second group & Lower bound & Difference of means & Upper bound \\
\hline \multirow{4}{*}{} & Hexane-capillary & Methanol/ammonia & -0.61 & -0.23 & 0.16 \\
& CFA & Hexane-packed & -0.65 & -0.16 & 0.34 \\
$*^{\mathrm{a}}$ & CFA & Methanol/ammonia & 0.13 & 0.53 & 0.92 \\
$*$ & CFA & Hexane-capillary & 0.48 & 0.76 & 1.03 \\
$*$ & CFA & MTBE & 0.79 & 1.08 & 1.36 \\
$*$ & Hexane-capillary & MTBE & 0.05 & 0.32 & 0.59 \\
$*$ & Hexane-capillary & Hexane-packed & -1.40 & -0.91 & -0.42 \\
$*$ & Hexane-packed & Methanol/ammonia & 0.12 & 0.68 & 1.25 \\
$*$ & Hexane-packed & MTBE & 0.73 & 1.23 & 1.73 \\
$*$ & MTBE & Methanol/ammonia & -0.94 & -0.55 & -0.16 \\
\hline
\end{tabular}

${ }^{a}$ An asterisk $\left({ }^{*}\right)$ indicates statistically significant comparisons (i.e., zero not included in the range spanned by the lower and upper bound).

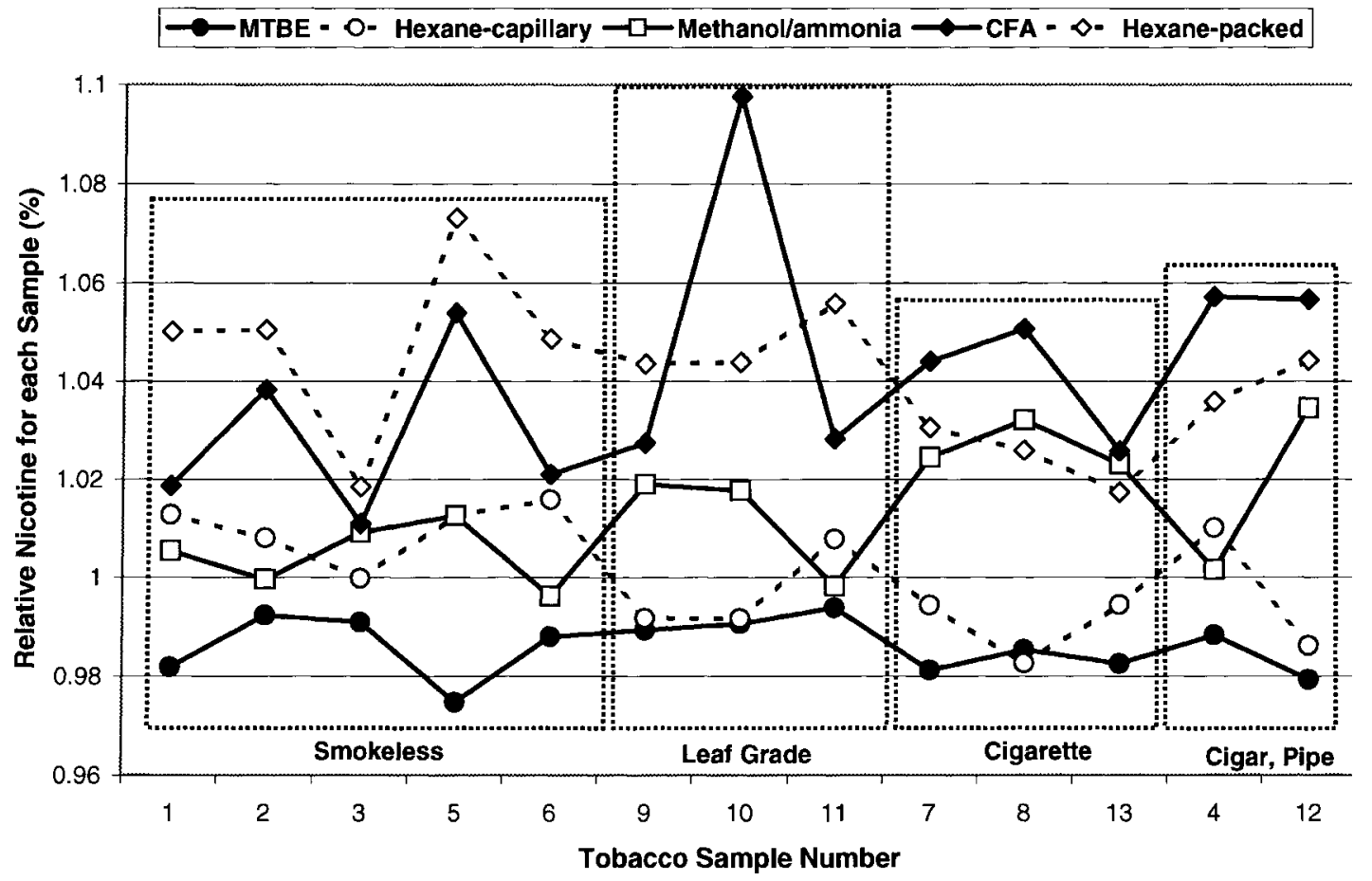

Figure 2.

Relative nicotine by sample type (the average of the three capillary GC methods is defined as $100 \%$ )

intercept (rather than best-fit line) is simply used to estimate $s_{r}$ or $s_{R}$, respectively. The best-fit intercept to a set of data is simply the average value of the data. Therefore, if there is no correlation of $s_{r j}$ with $m_{\mathrm{Nic}}$, then $s_{r}$ is given by $b_{r}=(1 / q) \sum s_{r j}$, where $s_{r j}$ is the repeatability of the $j^{\text {th }}$ sample and $q$ is the number of samples. Similarly, if there is no correlation of $s_{R j}$ with $m_{\mathrm{Nic}}$, then $s_{R}$ is given by $b_{R}=(1 / q) \sum s_{R j}$.

Whether $s_{r}$ or $s_{R}$ should be used to estimate the precision of any given nicotine measurement depends on the experimental conditions. In most cases, reproducibility conditions should be expected, in which both random experi- mental errors (e.g., repeatability errors) and errors resulting from different operators, instruments, laboratories, and effects of time may all influence the measurement. Since sample type was also varied in the collaborative study protocol and reproducibility statistics will include all sources of variance caused by variations in sample type, $s_{R}$ would also be expected to give a better estimate of the precision of a given nicotine method over various types of samples.

Since reproducibility statistics include all sources of precision errors, the relationship between $s_{R}$ and $m_{\mathrm{Nic}}$ is most useful in comparing the variance characteristics of 


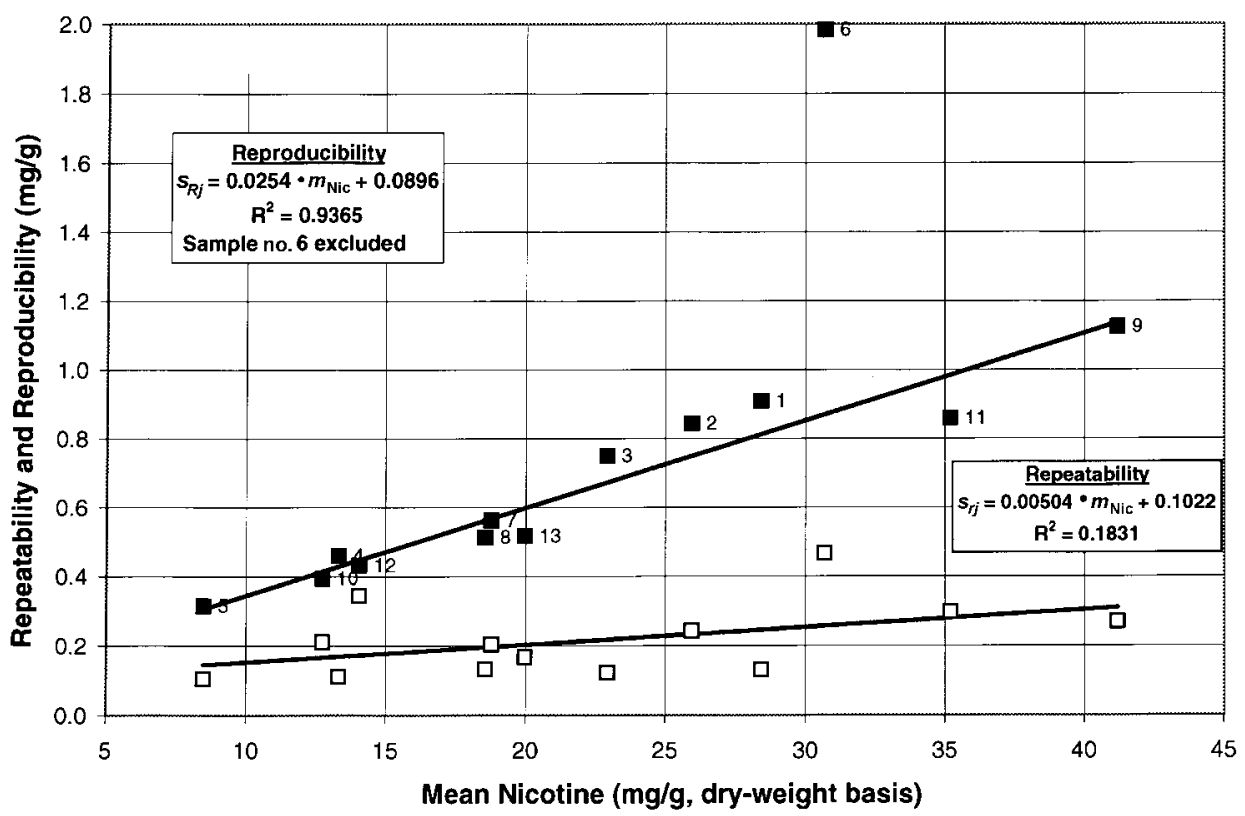

Figure 3 .

Dependence of $s_{r j}$ and $s_{R j}$ on mean nicotine $\left(m_{\mathrm{Nic}}\right)$ for the MTBE method using weighted least squares regression

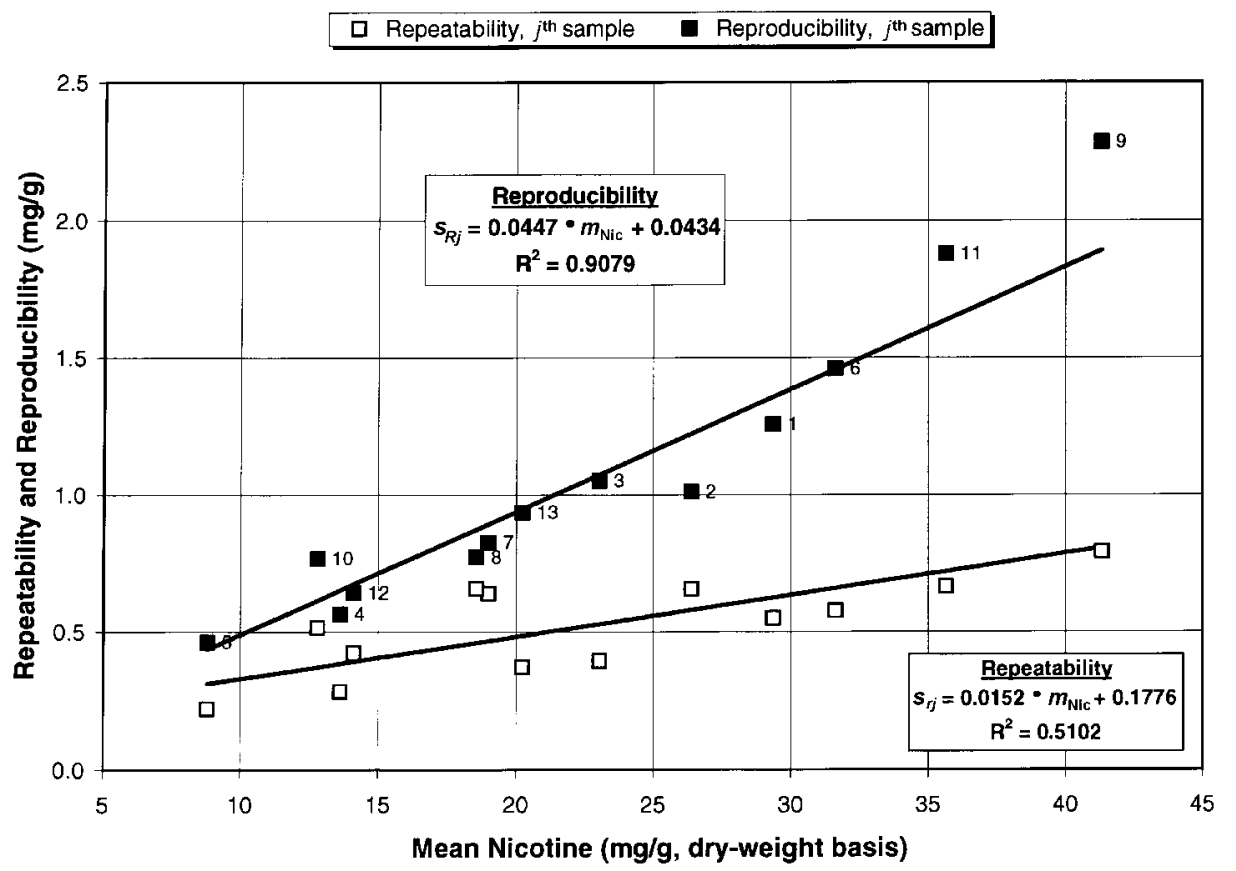

Figure 4.

Dependence of $s_{r j}$ and $s_{R j}$ on mean nicotine $\left(m_{\mathrm{Nic}}\right)$ for the hexane-capillary method using weighted least squares regression

the five nicotine methods. The lack of a functional (correlative) relationship between $s_{R}$ and $m_{\mathrm{Nic}}$ may indicate that sample heterogeneities exist that are not a regular function of $m_{\mathrm{Nic}}$. In the collaborative study, in which several types of tobacco samples were analyzed, differences in matrix effects from sample to sample may influence the reproducibility variance characteristics. As a result, one or a few nicotine samples might not follow a general relationship between $s_{R}$ and $m_{\mathrm{Nic}}$. If too many samples have different variance characteristics, it may be found that no satisfactory functional relationship is observed in a plot of $s_{R} v s . m_{\mathrm{Nic}}$. In this latter case, the average reproducibility is calculated to estimate $s_{R}$ for each method, as described above.

Figures 3-7 show plots of $s_{r} v s . m_{\mathrm{Nic}}$ and $s_{R}$ and $m_{\mathrm{Nic}}$ for the MTBE, hexane-capillary, methanol/ammonia, CFA, and hexane-packed methods, respectively. All of the regression equations in Figures 3-7 for repeatability and reproducibility for each method were determined using an iterative weighted least squares procedure (7). The $R^{2}$ 


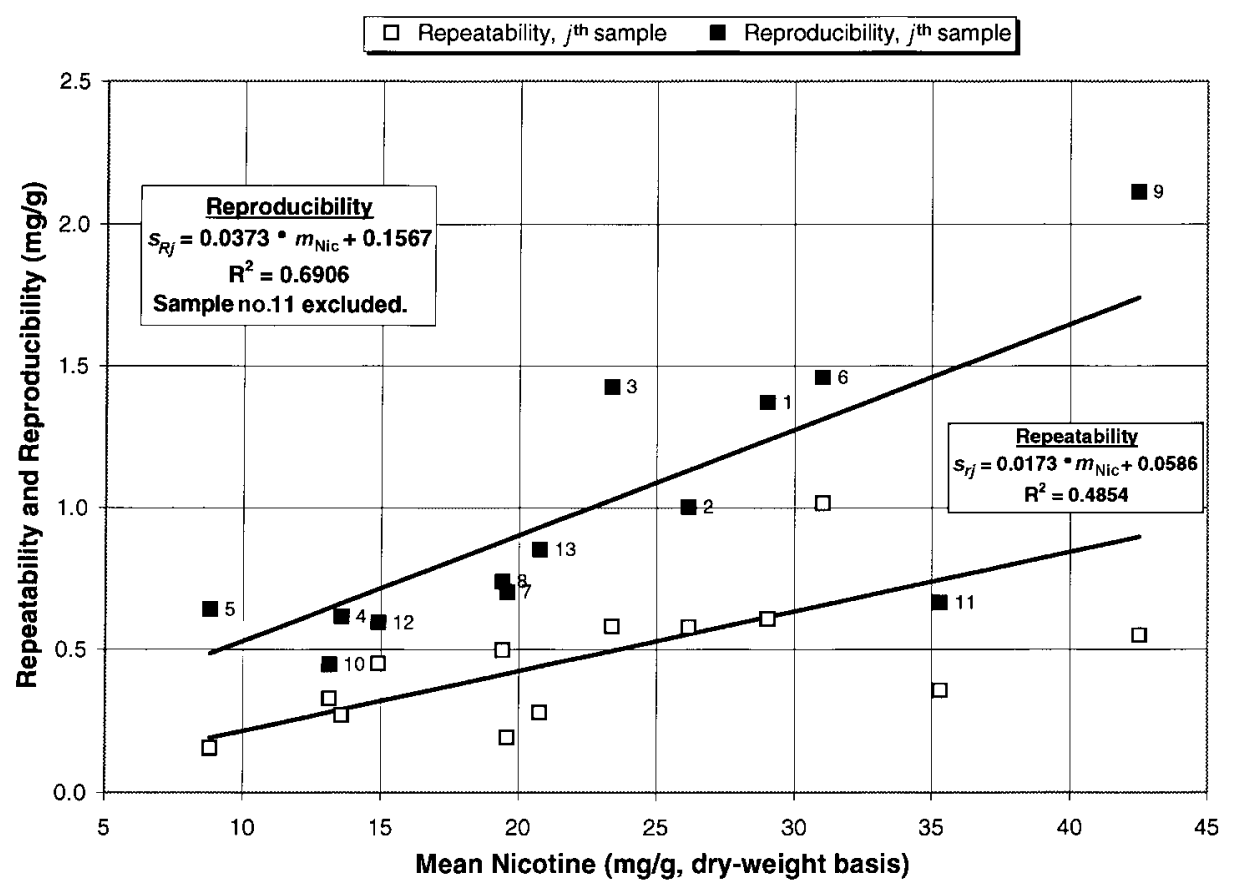

Figure 5.

Dependence of $s_{r j}$ and $s_{R j}$ on mean nicotine $\left(m_{\mathrm{Nic}}\right)$ for the methanol/ammonia method using weighted least squares regression

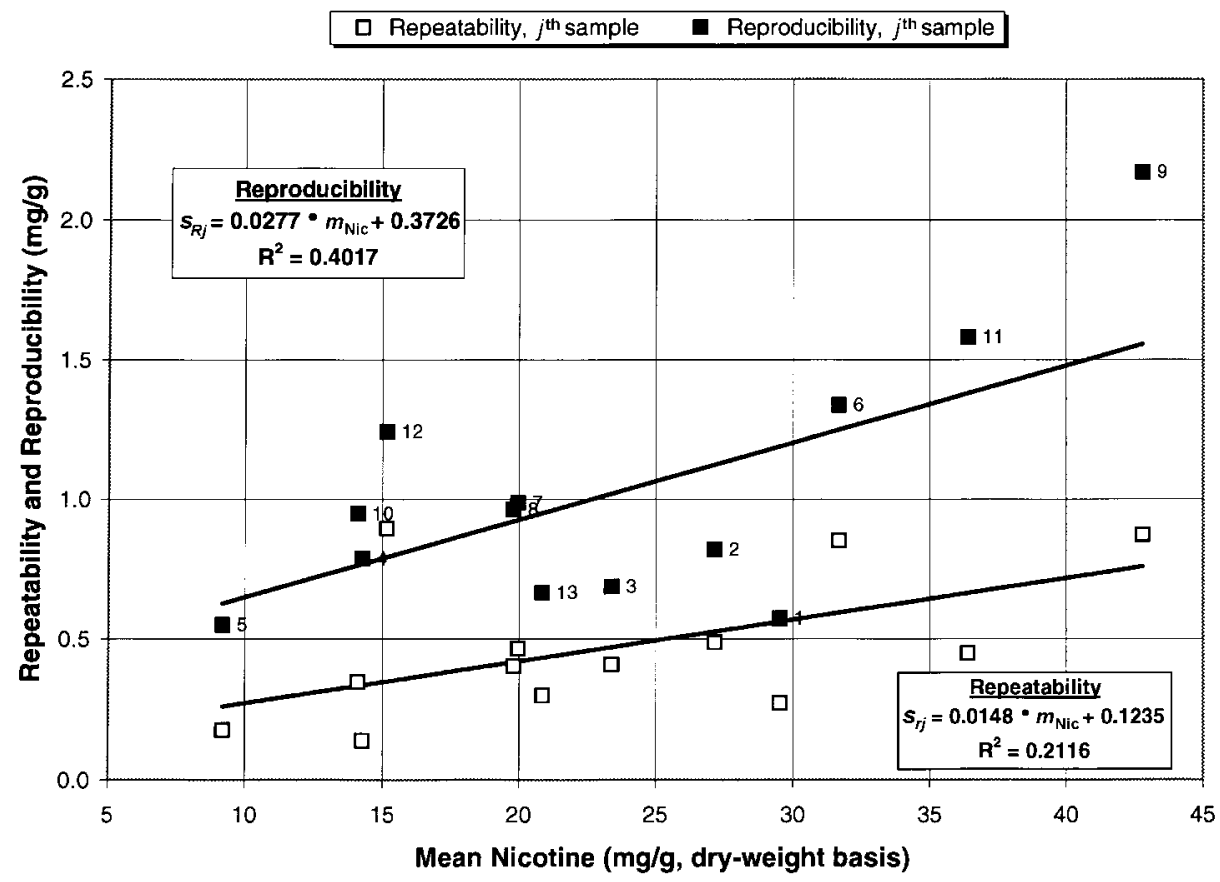

Figure 6.

Dependence of $s_{r j}$ and $s_{R j}$ on mean nicotine $\left(m_{\mathrm{Nic}}\right)$ for the CFA method using weighted least squares regression

values in the figures are weighted squared correlation coefficients, given by $R^{2}=1-\left(\mathrm{SSE}_{w} / \mathrm{SST}_{w}\right)$. For repeatability (or for reproducibility replace $r$ subscripts with $R$ subscripts in what follows), $\mathrm{SSE}_{w}=\sum w_{j}\left(s_{r j}-\hat{s}_{r j}\right)^{2}$, $\hat{s}_{r j}=\hat{b}_{w, r}+\hat{m}_{w, r} m_{\mathrm{Nic}}$, which is the weighted sum of squared errors, and $\mathrm{SST}_{w}=\sum w_{j}\left(s_{r j}-\bar{s}_{r}\right)^{2}, \bar{s}_{r}=\sum\left(w s_{r j}\right) / \sum w_{j}$, which is the weighted total sum of squares. None of the five methods produced a significant functional relationship between $s_{r}$ and $m_{\mathrm{Nic}}$, the weighted $R^{2}$ values varying between 0.18 and 0.51 . Thus, $b_{r}$ for each method was the reported $s_{r}$ for each method. Four methods showed good correlation between $s_{R}$ and $m_{\text {Nic }}$ (weighted $R^{2}$ between 0.69 and 0.94 ), so the respective $\hat{m}_{w, R}$ calculated for each of these four methods was the reported $s_{R}$ for the four methods. The CFA method exhibited poor correlation with $m_{\text {Nic }}$ (weighted $R^{2}$ of 0.40 ), so $b_{R}$ calculated from the CFA data was the reported $s_{R}$ for this method. 


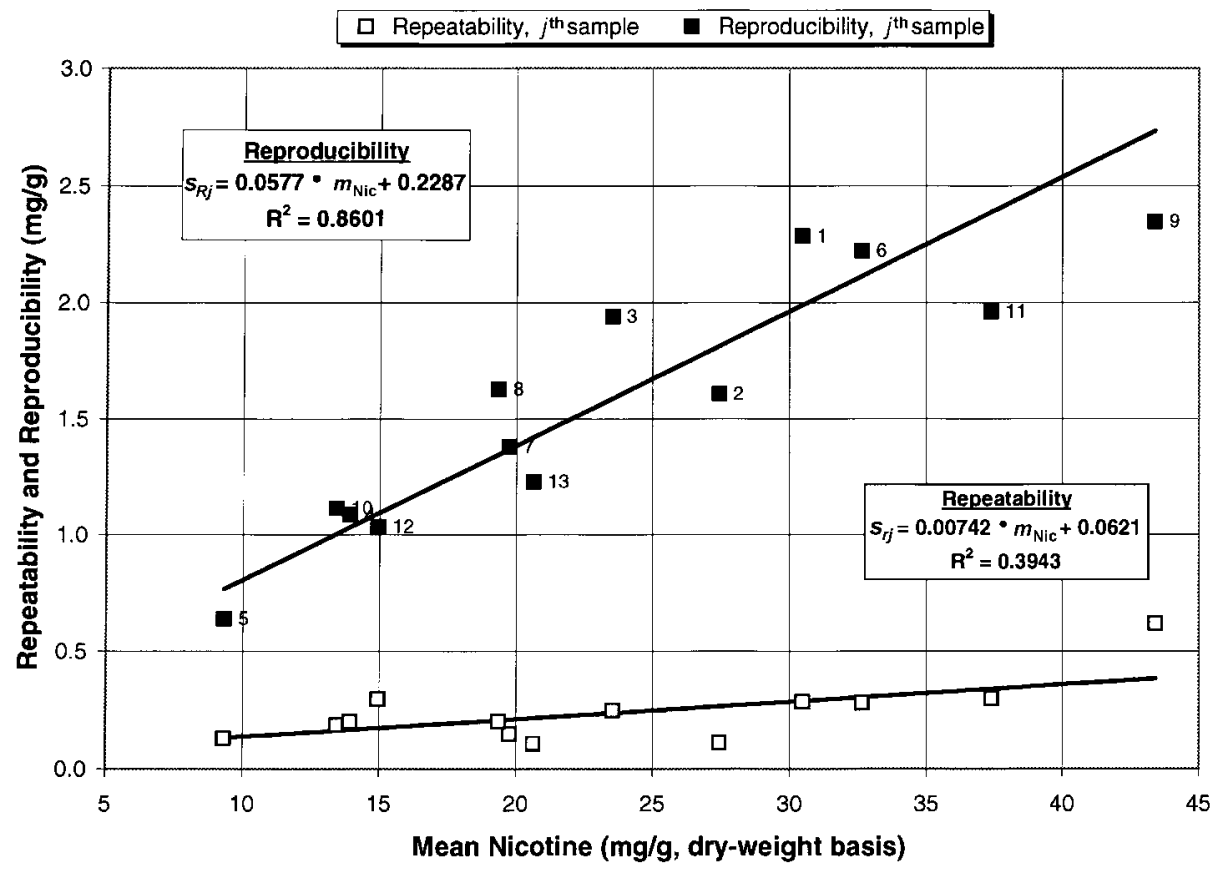

Figure 7.

Dependence of $s_{r j}$ and $s_{R j}$ on mean nicotine $\left(m_{\mathrm{Nic}}\right)$ for the hexane-packed method using weighted least squares regression

\section{Comparison of methods using repeatability and reproducibility statistics}

The repeatability and reproducibility statistics for each of the 13 samples and each of the five methods are given in Tables 5 and 6, respectively. The $b_{r}$ and $b_{R}$ values for each method in the tables are the average repeatability and reproducibility, as defined in the previous section. The $\% b_{r}$ and $\% b_{R}$ values are obtained by dividing $b_{r}$ and $b_{R}$, respectively, by the global average nicotine of all samples for a given nicotine method. In Table $6, \% \hat{m}_{w, R}$ for each method is $\hat{m}_{w, R}$ as a percentage. The overall repeatability and reproducibility for each nicotine method are summarized in Table 7 , reporting the slope values from Figures 3-7 only when the correlation was deemed significant. Figure 1 also graphically compares each of the five nicotine methods on the basis of their mean $\%$ difference, $\%$ repeatability, and $\%$ reproducibility. The \% standard error on the relative mean (\%SEM) is also shown for each method as the rightmost bar for each method and as an error bar on the mean \% difference bar. The \%SEM is calculated by dividing the reproducibility of each method by the square root of the number of samples, 13 , analyzed by each method and multiplying this quantity by the relative mean. The \%SEM is not a confidence interval on the relative mean, but it is useful as a visual aid in comparing the precision of any two or more of the five methods. Statistically meaningful comparisons of any two methods must be done using simultaneous confidence intervals and pair-wise group comparisons as described earlier and shown in Table 4.

Table 7 and Figure 1 show that the MTBE method yields the best reproducibility $(2.5 \%)$, followed by the methanol/ammonia (3.7\%), CFA (4.4\%), hexane-capillary
(4.5\%), and hexane-packed (5.8\%) methods. The MTBE method also produces the most repeatable nicotine measurements $(0.96 \%)$. For the methanol/ammonia and MTBE methods, one of the 13 tobacco samples was excluded in the determination of reproducibility precision for these two methods (see Figures 3 and 5). As mentioned earlier, the sample matrix is not constant in the collaborative study experimental design, which means that some samples may not follow the general trend in reproducibility observed for a given method. This secondary effect of the sample matrix is one possible explanation for why there exists one outlier point in the reproducibility plots for each of the MTBE and methanol/ammonia methods.

The fact that the three capillary GC methods yielded functional relationships of reproducibility with mean nicotine with good precision is meaningful in the context of deciding what methods are most self-consistent, and therefore more reliable, in the determination of nicotine in a wide variety of tobacco and tobacco products. Absent effects from sample heterogeneity, sample matrix, and method-specific interferences, any given nicotine method is be expected to exhibit a linear relationship of $s_{R}$ with $m_{\text {Nic }}$. The CFA and hexane-packed methods show less reliability than the three GC capillary methods due to lack of a functional relationship between $s_{R}$ and $m_{\mathrm{Nic}}$ (CFA) or poor reproducibility (hexane-packed). That these two methods were less reliable was also suggested when the mean nicotine values of the five methods were compared and these two methods showed a positive bias of about $4 \%$ relative to the three capillary GC methods. The reproducibility values determined in this collaborative study are reasonable in magnitude, based on a relationship between precision and concentration for col- 
Table 5.

Repeatability of nicotine determination ( $\mathrm{mg} / \mathrm{g}$, dry basis) by sample $\left(s_{r j}\right)$ for each method ${ }^{\mathrm{a}}$

\begin{tabular}{l|c|c|c|c|c}
\hline \multirow{2}{*}{ Sample } & \multicolumn{5}{c}{ Repeatability, $s_{r j}$} \\
\cline { 2 - 6 } & MTBE & $\begin{array}{c}\text { Hexane- } \\
\text { capillary }\end{array}$ & $\begin{array}{c}\text { Methanol/ } \\
\text { ammonia }\end{array}$ & CFA & $\begin{array}{c}\text { Hexane- } \\
\text { packed }\end{array}$ \\
\hline 1 & 0.13 & 0.55 & 0.61 & 0.27 & 0.28 \\
2 & 0.24 & 0.65 & 0.58 & 0.49 & 0.11 \\
3 & 0.12 & 0.40 & 0.58 & 0.41 & 0.25 \\
4 & 0.11 & 0.28 & 0.27 & 0.14 & 0.20 \\
5 & 0.10 & 0.22 & 0.15 & 0.18 & 0.13 \\
6 & 0.47 & 0.57 & 1.01 & 0.85 & 0.28 \\
7 & 0.20 & 0.64 & 0.19 & 0.47 & 0.15 \\
8 & 0.13 & 0.66 & 0.50 & 0.40 & 0.20 \\
9 & 0.27 & 0.79 & 0.55 & 0.87 & 0.62 \\
10 & 0.21 & 0.51 & 0.33 & 0.34 & 0.18 \\
11 & 0.30 & 0.66 & 0.36 & 0.45 & 0.30 \\
12 & 0.34 & 0.42 & 0.45 & 0.89 & 0.30 \\
13 & 0.17 & 0.37 & 0.28 & 0.30 & 0.11 \\
$b_{r}$ & 0.21 & 0.52 & 0.45 & 0.47 & 0.24 \\
$\% b_{r}$ & $0.96 \%$ & $2.29 \%$ & $1.96 \%$ & $1.99 \%$ & $1.01 \%$ \\
\hline
\end{tabular}

${ }^{\text {aT }}$ The $b_{r}$ values represent the average of the $s_{r j}$ values for each method over all samples. The $\% b_{r}$ values are given by $b_{r}$ divided by the average nicotine of all samples for each method.

laborative studies reported in the literature. BOYER et al. (10) examined the results of numerous collaborative studies that spanned a wide range of analyte concentrations (ppb to few \%) and a wide range of sample matrices (drug formulations, feeds, pesticide residues, etc.). They discuss an empirical equation relating the relative standard deviation of reproducibility, $\mathrm{RSD}_{R}(\%)$, and the sample concentration, $C$, which is expressed in equation [1].

$$
\operatorname{RSD}_{R} \%=2^{\left(1-0.5 \cdot \log _{10} C\right)} \text {. }
$$

In equation [1], $C$ must be expressed as a decimal fraction (e.g., $g / g$ basis). Based on the consistency with which collaborative study data obey the empirical relationship above, the authors state that the applicability of a method to determine an analyte in a given matrix should be questioned if its $\mathrm{RSD}_{R}$ exceeds twice the value predicted by equation [1]. In the nicotine collaborative study, nicotine varies from 9 to $43 \mathrm{mg} / \mathrm{g}$, or 0.009 to 0.043 as a decimal fraction. Equation [1] predicts that $\mathrm{RSD}_{R}(\%)$ should be $3.2 \%$ to $4.1 \%$ at the low and high levels, respectively, of nicotine among the 13 tobacco samples. The $\mathrm{RSD}_{R}(\%)$ estimates from equation [1] are indeed similar to the reproducibility statistics determined for any of the nicotine methods, which range between $2.5 \%$ to $5.8 \%$; and none of the methods exceed twice the $\mathrm{RSD}_{R}(6.4-8.2 \%)$ predicted by equation [1]. The similarity of the observed reproducibility statistics of all nicotine methods with $\mathrm{RSD}_{R}(\%)$ predicted from the literature suggests that the
Table 6.

Reproducibility of nicotine determination ( $\mathrm{mg} / \mathrm{g}$, dry basis) by sample $\left(s_{R j}\right)$ for each method ${ }^{\mathrm{a}}$

\begin{tabular}{|c|c|c|c|c|c|}
\hline \multirow[b]{2}{*}{ Sample } & \multicolumn{5}{|c|}{ Reproducibility, $s_{R j}$} \\
\hline & MTBE & $\begin{array}{l}\text { Hexane- } \\
\text { capillary }\end{array}$ & $\begin{array}{l}\text { Methanol/ } \\
\text { Ammonia }\end{array}$ & CFA & $\begin{array}{l}\text { Hexane- } \\
\text { packed }\end{array}$ \\
\hline 1 & 0.91 & 1.26 & 1.37 & 0.57 & 2.28 \\
\hline 2 & 0.84 & 1.01 & 1.00 & 0.82 & 1.61 \\
\hline 3 & 0.75 & 1.05 & 1.42 & 0.69 & 1.94 \\
\hline 4 & 0.46 & 0.56 & 0.61 & 0.79 & 1.09 \\
\hline 5 & 0.31 & 0.46 & 0.64 & 0.55 & 0.64 \\
\hline 6 & 1.98 & 1.45 & 1.45 & 1.34 & 2.21 \\
\hline 7 & 0.56 & 0.83 & 0.70 & 0.99 & 1.38 \\
\hline 8 & 0.51 & 0.77 & 0.74 & 0.96 & 1.62 \\
\hline 9 & 1.12 & 2.28 & 2.11 & 2.17 & 2.35 \\
\hline 10 & 0.39 & 0.76 & 0.44 & 0.95 & 1.11 \\
\hline 11 & 0.86 & 1.88 & 0.66 & 1.58 & 1.96 \\
\hline 12 & 0.43 & 0.64 & 0.59 & 1.24 & 1.04 \\
\hline 13 & 0.52 & 0.93 & 0.85 & 0.66 & 1.23 \\
\hline$b_{R}$ & 0.74 & 1.07 & 0.97 & 1.02 & 1.57 \\
\hline$\% b_{R}$ & $3.32 \%$ & $4.72 \%$ & $4.24 \%$ & $4.37 \%$ & $6.68 \%$ \\
\hline$\% \hat{m}_{w, R}$ & $2.54 \%$ & $4.47 \%$ & $3.73 \%$ & $2.77 \%$ & $5.77 \%$ \\
\hline
\end{tabular}

aThe $b_{R}$ values represent the average of the $s_{R j}$ values for each method over all samples. The $\% b_{R}$ values are given by $b_{R}$ divided by the average nicotine of all samples for each method. The $\% \hat{m}_{w, R}$ values are given by the slope (as a percentage) of the weighted least-squares line fit to the $s_{R j} v s . m_{\mathrm{Nic}}$ data for each nicotine method (see Figures 3-7).

collaborative study data are of good quality and that analysis procedures, such as outlier removal, were adequate and appropriate.

\section{CONCLUSIONS}

Comparing methods on an individual basis, the MTBE method possessed the lowest mean nicotine values on average and the best reproducibility of all methods evaluated. The closest equivalent method, hexane-capillary, yielded reproducibility that was $80 \%$ larger than that of the MTBE method. The hexane-packed method yielded the highest mean nicotine values and the worst reproducibility of any of the five methods evaluated.

Grouping methods based on consistency, it is clear that the mean nicotine values for the three capillary GC methods are similar, with the maximum mean difference being 2.7\% between the methanol/ammonia and MTBE methods. The mean nicotine values of the CFA and hexane-packed methods are positively biased by about $4 \%$ relative to the mean nicotine values of the three capillary GC methods. The CFA methods also produced the weakest linear relationship between reproducibility and mean nicotine and the hexane-packed method had the worst reproducibility. These facts support the conclusion 
Table 7.

Summary of results for the nicotine collaborative study

\begin{tabular}{lcc|c|c|c}
\hline & MTBE & Hexane-capillary & $\begin{array}{c}\text { Methanol/ } \\
\text { ammonia }\end{array}$ & CFA & Hexane-packed \\
\hline Mean difference $^{\mathrm{a}, \mathrm{d}}$ & $-0.31(-1.40 \%)$ & $0.01(0.06 \%)$ & $0.31(1.34 \%)$ & $0.96(4.08 \%)$ & $0.98(4.14 \%)$ \\
$s_{r}\left(\right.$ Repeatability) $^{\mathrm{a}}$ & $0.21(1.0 \%)^{\mathrm{b}}$ & $0.52(2.3 \%)^{\mathrm{b}}$ & $0.45(2.0 \%)^{\mathrm{b}}$ & $0.47(2.0 \%)^{\mathrm{b}}$ & $0.24(1.0 \%)^{\mathrm{b}}$ \\
$s_{R}$ (Reproducibility) $^{\mathrm{a}}$ & $0.57(2.5 \%)^{\mathrm{c}}$ & $1.01(4.5 \%)^{\mathrm{c}}$ & $0.85(3.7 \%)^{\mathrm{c}}$ & $1.02(4.4 \%)^{\mathrm{b}}$ & $1.36(5.8 \%)^{\mathrm{c}}$ \\
Ratio $^{\mathrm{d}}$ & $98.6 \%$ & $100.1 \%$ & $101.3 \%$ & $104.1 \%$ & $104.1 \%$ \\
$\%$ Outliers $_{\text {Mean } \% \text { diff. stdev }}$ & $16.3 \%$ & $6.6 \%$ & $12.8 \%$ & $16.8 \%$ & $18.0 \%$ \\
Lower bound & $0.69 \%$ & $1.2 \%$ & $1.0 \%$ & $1.3 \%$ & $1.7 \%$ \\
Upper bound & $97.9 \%$ & $98.8 \%$ & $100.3 \%$ & $102.8 \%$ & $102.5 \%$ \\
Number of labs & $99.3 \%$ & $101.3 \%$ & $102.4 \%$ & $105.3 \%$ & $105.8 \%$ \\
\hline
\end{tabular}

aUnits are $\mathrm{mg} / \mathrm{g}$ (dry-weight basis).

${ }^{\mathrm{b}}$ Derived from $b_{r}$ or $b_{R}$ (average $s_{r}$ or $s_{R}$ calculation).

'Derived from slope of weighted least squares regression of $s_{R}$ on $m$.

${ }^{\mathrm{d}}$ Relative to mean nicotine derived from the three GC-capillary methods.

that the CFA and hexane-packed methods are the least reliable methods for the determination of nicotine over a wide variety of tobacco and tobacco products. In contrast, the three capillary GC methods show the most reliability based upon their similarity in mean nicotine values and the fact that these three methods produced satisfactory linear relationships between reproducibility and mean nicotine.

The collaborative study was successful in meeting its objectives to compare precision and to evaluate agreement among the five nicotine methods. The most important observation from the study is the consistency of the three capillary GC methods in terms of mean nicotine and variance characteristics. This observation supports the conclusion that a capillary GC method can be chosen with a high degree of confidence as a reference method for the determination of nicotine over a wide variety of tobacco and tobacco products.

The reproducibility precision of all five nicotine methods ( $2.5 \%$ to $5.8 \%$ ) conform to the reproducibility predicted by the empirical Horwitz equation (3.2\% to $4.1 \%$, with confidence intervals of $6.4 \%$ to $8.2 \%$ ) for the range of nicotine values spanned by the 13 tobacco samples in the collaborative study. This agreement between the observed reproducibility values in the current study and the reproducibility values predicted from an empirical relationship derived by observing the results of a large number of collaborative studies suggest that the data and results from the nicotine collaborative study are of good quality and that appropriate data analysis procedures were applied. Further refinement of the collaborative protocol, including uniform standards for all methods and more detailed instructions to participants to achieve higher compliance, would be expected to yield fewer outlier samples, better reproducibility results, and improved agreement among the capillary GC methods.

\section{REFERENCES}

1. Borgerding, M.F., T.A. Perfetti, and S. Ralapati: Determination of nicotine in tobacco, tobacco processing environments and tobacco products; in: Analytical determination of nicotine and related compounds and their metabolites, edited by J.W. Gorrod and P. Jacob, III, Elsevier, Amsterdam, Ch. 9, 1999, pp. 285-391.

2. Federal Register, v. 64, no. 55, March 23, 1999.

3. CORESTA recommended method no. 35: Determination of total alkaloids in tobacco by continuous flow analysis; final approval, Nov. 1994, Cooperation Centre for Scientific Research relative to tobacco, CORESTA Inf. Bulletin 1994 (3/4) 39-46.

4. Bennett, C.B., United States Tobacco Manufacturing LP, personal communication, May 1999.

5. Hahn, J., Chemische Landesuntersuchungsanstalt Sigmaringen, personal communication, May 1999.

6. Davis, R.E., Philip Morris Research Center, personal communication, May 1999.

7. ISO 5725-2:1994(E): Accuracy (trueness and precision) of measurement methods and results - Part 2: Basic method for the determination of repeatability and reproducibility of a standard measurement method, International Organization for Standardization, Geneva, 1994.

8. AOAC official methods program: policies and procedures manual: Association of Official Analytical Chemists (AOAC) International, Gaithersburg, Maryland, 1998, p. 3.

9. Jacob, III, P. and G.D. Byrd: Use of gas chromatographic and mass spectrometric techniques for the determination of nicotine and its metabolites; in: Analytical determination of nicotine and related compounds and their metabolites, edited by J.W. 
Gorrod and P. Jacob, III, Elsevier, Amsterdam, Ch. 6, 1999, pp. 191-224.

10. Boyer, K.W., W. Horwitz, and R. Albert: Interlaboratory variability in trace element analysis; Anal. Chem. 57 (1985) 454 459.

\section{Acknowledgments}

Mr. David Self is appreciated for his efforts in preparing the tobacco samples and for distribution to participants in the study. The authors are also grateful to Dr. Shawn $M$. Shanmugan for assistance in protocol development and method evaluation, to Dr. Michael F. Borgerding for helpful discussions during the evaluation phase, and to Dr. Harold Burton for helpful advice all along the way. Special gratitude must also be extended to the 37 laboratories that contributed their time and effort to provide data, specifically British American Tobacco (Germany) GmbH, British American Tobacco - R\&D Centre, Bundesinstitur für gesundheitlichen Verbraucherschutz und Veterinärmedizin, Brown \& Williamson Tobacco Corp., Chemische Landesuntersuchungsanstalt Sigmaringen, Compania Colombiana de Tabaco S.A., Ento Tabacchi Italiani, Filtrona, Gallaher Lid., Heinr. Borgwald GmbH, Heinz van Landewyck, Imperial Tobacco Ltd.,
Imperial Tobacco RYO BV, J.L. Tiedmanns Tobaksfabrik, Japan Tobacco Inc., Japan Tobacco Inc. (UK), Landesanstalt für Pflanzenbau (LAP), Laboratory of the Government Chemist, Labstat, LTR Industries, National Tobacco, Philip Morris Europe, Philip Morris Mexico, Philip Morris USA, H.F. \& PH.F. Reemtsma, Rothmans International, Rothmans International Europe b.v., R.J. Reynolds Tobacco, Rothmans of Pall Mall (International) Ltd., Koninklijke Theodorius Niemeyer B.V., SEITA - Centre de Recherche, Swisher International, Swedish Match, Swedish Match - North America, Tabacalera S.A., U.S. Tobacco Manufacturing LP, and W. E. J.

Address for correspondence

Cliff B. Bennett

U.S. Tobacco Manufacturing L. P.

Research and Development Department

800 Harrison Street

Nasbville, TN 37203 (USA)

Emailcbennett@ustha.com

\section{ERRATUM NOTICE}

p. 251: author statement (byline) should read:

"James E. Franke, Cliff B. Bennett, Richard E. Davis, Hans V. Thomsen, Kathleen S.

Johnston, and Shawn M. Shanmugan"

S.M. Shanmugan being a scientist with U.S.

Tobacco Manufacturing, L.P., Nashville, TN, USA. 